\title{
Detailed analysis of sputum and systemic inflammation in asthma phenotypes: are paucigranulocytic asthmatics really non-inflammatory?
}

Sophie Demarche ${ }^{1,2,3^{*}}$, Florence Schleich ${ }^{1,2}$, Monique Henket ${ }^{1,2}$, Virginie Paulus ${ }^{1,2}$, Thierry Van Hees ${ }^{3}$ and Renaud Louis ${ }^{1,2}$

\begin{abstract}
Background: The technique of induced sputum has allowed to subdivide asthma patients into inflammatory phenotypes according to their level of granulocyte airway infiltration. There are very few studies which looked at detailed sputum and blood cell counts in a large cohort of asthmatics divided into inflammatory phenotypes. The purpose of this study was to analyze sputum cell counts, blood leukocytes and systemic inflammatory markers in these phenotypes, and investigate how those groups compared with healthy subjects.

Methods: We conducted a retrospective cross-sectional study on 833 asthmatics recruited from the University Asthma Clinic of Liege and compared them with 194 healthy subjects. Asthmatics were classified into inflammatory phenotypes.

Results: The total non-squamous cell count per gram of sputum was greater in mixed granulocytic and neutrophilic phenotypes as compared to eosinophilic, paucigranulocytic asthma and healthy subjects $(p<0.005)$. Sputum eosinophils (in absolute values and percentages) were increased in all asthma phenotypes including paucigranulocytic asthma, compared to healthy subjects $(p<0.005)$. Eosinophilic asthma showed higher absolute sputum neutrophil and lymphocyte counts than healthy subjects $(p<0.005)$, while neutrophilic asthmatics had a particularly low number of sputum macrophages and epithelial cells. All asthma phenotypes showed an increased blood leukocyte count compared to healthy subjects $(p<0.005)$, with paucigranulocytic asthmatics having also increased absolute blood eosinophils compared to healthy subjects $(p<0.005)$. Neutrophilic asthma had raised CRP and fibrinogen while eosinophilic asthma only showed raised fibrinogen compared to healthy subjects $(p<0.005)$.

Conclusions: This study demonstrates that a significant eosinophilic inflammation is present across all categories of asthma, and that paucigranulocytic asthma may be seen as a low grade inflammatory disease.
\end{abstract}

Keywords: Asthma, Phenotypes, Healthy subjects, Sputum cytology, Blood leukocyte count, CRP, Fibrinogen

\footnotetext{
* Correspondence: sophie.demarche@ulg.ac.be

${ }^{1}$ Department of Respiratory Medicine, CHU Liege, Sart-Tilman B35, 4000

Liege, Belgium

${ }^{2}$ GIGA I Research Group, University of Liege, Liege, Belgium

Full list of author information is available at the end of the article
} 


\section{Background}

The technique of induced sputum has been pivotal in the emergence of the concept of inflammatory asthma phenotypes. Although it is technically demanding and time-consuming, several centers have applied the technique of induced sputum to characterize asthma inflammatory phenotypes in routine [1-7]. It has been suggested that airway inflammation may be subdivided into four phenotypes according to the level of granulocyte airway infiltration: eosinophilic, neutrophilic, mixed granulocytic and paucigranulocytic [2, 3]. This latter has been considered as a non-inflammatory type of asthma as the sputum analysis of these patients was suggested not to differ from healthy subjects [8].

These inflammatory phenotypes have been analyzed with respect to their demographic, functional and clinical characteristics in several studies $[2,3,9,10]$. However, there has been fewer studies looking in detail at sputum cells [2], blood leukocytes [11], systemic inflammatory markers or at these variables in combination in a large cohort of asthmatics classified according to the extent of airway granulocytic inflammation, and investigating how those groups compared with healthy subjects.

Here, in a retrospective analysis, we report on sputum cell counts, blood leukocytes, C-reactive protein (CRP) and fibrinogen in a large series of asthmatics seen in daily practice of a University Clinic and compare the results with those in healthy subjects.

Our results show that paucigranulocytic asthmatics may display a low grade airway and systemic inflammation.

\section{Methods}

\section{Study design, setting and participants}

We conducted a retrospective cross-sectional study on asthmatic patients and healthy subjects recruited from the University Asthma Clinic of Liege. Asthmatic patients were eligible for the study if they had a first visit with a successful sputum analysis between 1 October 2003 and 5 February 2015. The diagnosis of asthma was based on the presence of typical symptoms (wheezing, breathlessness, chest tightness, cough) and at least one of the following: forced expiratory volume in one second $\left(\mathrm{FEV}_{1}\right)$ increase of $\geq 12 \%$ and $200 \mathrm{ml}$ after inhalation of $400 \mu \mathrm{g}$ salbutamol or a provocative concentration of methacholine causing a $20 \%$ fall in $\mathrm{FEV}_{1}$ (PC20M) less than $16 \mathrm{mg} / \mathrm{ml}$. Atopy was defined by the presence of at least one positive specific IgE (>0.35 kU/L; Phadia; Groot-Bijgaarden, Belgium) to one or more common aeroallergen (cat, dog, grass pollen, tree pollen, house dust mite and a mixture of moulds). Fractional exhaled nitric oxide (FENO) measurements were performed at $50 \mathrm{ml} / \mathrm{s}$ of flow rate (NIOX, Aerocrine, Sweden). Healthy subjects were recruited by advertisement and in the hospital staff, during this 12-year period of study. They had no diagnosis of respiratory disease, a $\mathrm{FEV}_{1} \geq 80 \%$ and a Tiffeneau index $\geq 0.7$. Only healthy subjects with a successful sputum analysis were selected. This retrospective study was conducted with the approval from the ethics committee of the University Hospital of Liege (Reference 2015/193). Informed consents were obtained from healthy subjects. As for asthmatic patients, all procedures were performed in the context of clinical practice and the retrospective data collection was conducted with the approval from the above-mentioned ethics committee.

\section{Sputum induction and analysis}

Sputum was induced and processed as previously described [12]. Briefly, sputum was induced by hypertonic (5\%) or isotonic saline with salbutamol when post-bronchodilator $\mathrm{FEV}_{1}$ was $>65 \%$ or $\leq 65 \%$ predicted, respectively. Sputum was then processed using the whole expectorate technique. Dithiothreitol (DTT) was used as the mucolytic agent. Sputum sample was considered as adequate for cell count when squamous cell count was $<80 \%$ [13]. Differential cell count was performed on cytospins after a Diff-Quick staining.

\section{Asthma phenotypes}

Threshold values used to define the eosinophilic and neutrophilic phenotypes were a sputum eosinophil count $\geq 3 \%$ and a sputum neutrophil count $\geq 76 \%$, respectively [14]. The mixed granulocytic phenotype was defined as both raised sputum eosinophil and neutrophil counts and the paucigranulocytic one as sputum eosinophil and neutrophil counts lower than the thresholds.

\section{Statistical analysis}

Results were expressed as frequencies and percentages for categorical variables and as median (interquartile range) or mean \pm standard deviation for continuous variables. Comparisons were performed using a Pearson's chi-squared test for categorical variables, an ANOVA or a Student's $t$-test for parametric variables, and a KruskalWallis test for non-parametric variables. The Pearson correlation coefficient was used to measure the association between the percentage of sputum neutrophils and eosinophils, and the number of pack-years in asthmatics. For this parametric test, sputum eosinophils were logtransformed and the 0 values replaced by 0.1 . A $p$ value $<0.05$ was considered statistically significant. When multiple tests were performed, the statistically significant level was corrected according to the Bonferroni principle. Statistical analysis was done using STATA version 13.0 (Statistical Software, College Station, TX: StataCorp LP). 


\section{Results}

\section{Subject characteristics}

The demographic, functional and treatment characteristics of asthmatics and healthy subjects are given in Table 1. In our cohort of asthmatics with a successful sputum analysis $(n=833)$, inflammatory phenotypes were distributed as follows: $42 \%$ of patients were eosinophilic, $16 \%$ neutrophilic, $4 \%$ mixed granulocytic and $38 \%$ paucigranulocytic. The group of neutrophilic asthma presented a higher proportion of women while eosinophilic asthma had a lower proportion of women. Paucigranulocytic asthmatics were the youngest and had the best $\mathrm{FEV}_{1}$ (\% predicted) and $\mathrm{FEV}_{1} /$ forced vital capacity (FVC) ratio. All asthma subgroups had a higher prevalence of atopy as compared to healthy subjects $(p<0.005$ : level of statistical significance after Bonferroni correction).

\section{Sputum cell counts and viability}

The sputum cell counts and viability of asthma phenotypes and healthy subjects are shown in Table 2 . The total non-squamous cell count per gram of sputum and the cell viability were greater in mixed granulocytic and neutrophilic phenotypes compared to eosinophilic, paucigranulocytic asthma and healthy subjects. Eosinophilic asthmatics also had significantly greater total sputum cell counts than paucigranulocytic asthmatics and healthy subjects. The fraction of squamous cells was less than $20 \%$ in a majority of the patients, and was particularly low in eosinophilic, neutrophilic and mixed granulocytic asthma. While levels were obviously the highest in eosinophilic and mixed granulocytic phenotypes compared to other asthmatics, sputum eosinophils (in absolute values and percentages) were increased in all asthma phenotypes, compared to healthy subjects (Table 2 and Fig. 1a). Absolute neutrophil counts were increased not only in neutrophilic and mixed granulocytic asthma, but also in eosinophilic asthma subjects, compared with healthy subjects. The group of neutrophilic asthma was the only one to have a lower absolute sputum macrophage count than eosinophilic, paucigranulocytic asthmatics and healthy subjects. Absolute sputum lymphocyte count was greater in eosinophilic asthma $(p<0.005$, versus paucigranulocytic asthma and healthy subjects), while absolute epithelial cell count was remarkably lower in case of intense neutrophilic inflammation $(p<0.005$, versus paucigranulocytic and eosinophilic asthma, Table 2 and Fig. 1b).

Table 1 Demographic, functional and treatment characteristics of asthmatics classified by phenotypes and healthy subjects

\begin{tabular}{|c|c|c|c|c|c|c|}
\hline & Eosinophilic asthma & Neutrophilic asthma & Mixed granulocytic asthma & Paucigranulocytic asthma & Healthy subjects & $p$ value \\
\hline N (\% of asthmatics) & $350(42)$ & $134(16)$ & $31(4)$ & $318(38)$ & 194 & - \\
\hline Women, N (\%) & $184(53)$ & $95(71)^{\neq \dagger}$ & $19(61)$ & $197(62)$ & $105(54)$ & 0.002 \\
\hline Age, years & $49(34-60)^{*}$ & $52(41-61)^{\ddagger^{*}}$ & $61(43-71)^{\ddagger^{*}}$ & $43(30-54)$ & $45(27-56)$ & 0.0001 \\
\hline BMl & $25.9 \pm 4.9^{\ddagger}$ & $25.3 \pm 5.4$ & $25.5 \pm 4.6$ & $26.1 \pm 4.8^{\ddagger}$ & $24.3 \pm 4.1$ & 0.0004 \\
\hline Atopy, N (\%) & $213(63)^{\ddagger}$ & $69(52)^{\ddagger}$ & $17(57)^{\ddagger}$ & $165(53)^{\ddagger}$ & $46(28)$ & $<0.001$ \\
\hline \multicolumn{7}{|l|}{ Smoking status, N (\%) } \\
\hline Non-smokers & $190(54)$ & $73(55)$ & $19(61)$ & $162(52)$ & $109(57)$ & $>0.05$ \\
\hline Current smokers & $64(18)$ & $26(20)$ & $5(16)$ & $76(24)$ & $35(18)$ & \\
\hline Ex-smokers & $96(27)$ & $33(25)$ & $7(23)$ & $75(24)$ & $47(25)$ & \\
\hline $\mathrm{FEV}_{1}, \%$ predicted & $81.2 \pm 20.9^{\ddagger^{*}}$ & $81.6 \pm 22.0^{\ddagger^{*}}$ & $81.6 \pm 20.6^{\ddagger}$ & $89.2 \pm 18.2^{\ddagger}$ & $108.0 \pm 13.3$ & 0.0001 \\
\hline $\mathrm{FEV}_{1} / \mathrm{FVC}, \%$ & $70.7 \pm 11.1^{キ^{*}}$ & $71.7 \pm 11.5^{\ddagger^{*}}$ & $70.1 \pm 10.5^{\ddagger^{*}}$ & $76.2 \pm 9.0^{\ddagger}$ & $82.1 \pm 6.5$ & 0.0001 \\
\hline FENO, ppb & $43(23-82)^{*}$ & $20(13-33)^{\dagger \S}$ & $36(21-75)^{*}$ & $16(12-29)$ & / & 0.0001 \\
\hline ICS therapy, N (\%) & $227(65)$ & $87(65)$ & $18(58)$ & $195(61)$ & / & $>0.05$ \\
\hline ICS dose $e^{a}$ & $500(0-1000)$ & $800(0-2000)$ & $800(0-1600)$ & $400(0-1000)$ & / & $>0.05$ \\
\hline OCS therapy, N (\%) & $21(6)$ & $9(7)$ & $2(6)$ & $14(4)$ & / & $>0.05$ \\
\hline LABA, N (\%) & $209(60)$ & $80(60)$ & $20(65)$ & $181(57)$ & / & $>0.05$ \\
\hline LTRA, N (\%) & $75(21)$ & $37(28)$ & $9(29)$ & $73(23)$ & / & $>0.05$ \\
\hline Theophylline, N (\%) & $11(3)$ & $7(5)$ & $0(0)$ & 11 (3) & / & $>0.05$ \\
\hline
\end{tabular}

${ }^{\ddagger} p<0.005$, comparison with healthy subjects

* $p<0.005$, comparison with paucigranulocytic asthma

${ }^{\dagger} p<0.005$, comparison with eosinophilic asthma

${ }^{5} p<0.005$, comparison with mixed granulocytic asthma

${ }^{\text {ICS }}$ dose in beclomethasone dipropionate - chlorofluorocarbon equivalents

Abbreviations: $B M I$ body mass index; FENO fractional exhaled nitric oxide; FEV forced expiratory volume in $1 \mathrm{~s}$; FVC forced vital capacity; ICS inhaled corticosteroid; $\angle A B A$ long-acting $\beta 2$-agonist; LTRA leukotriene receptor antagonist; OCS oral corticosteroid 
Table 2 Sputum cell counts and viability of asthmatics classified by phenotypes and healthy subjects

\begin{tabular}{|c|c|c|c|c|c|c|}
\hline & Eosinophilic asthma & Neutrophilic asthma & Mixed granulocytic asthma & Paucigranulocytic asthma & \multirow{3}{*}{$\begin{array}{l}\text { Healthy } \\
\text { subjects }\end{array}$} & \multirow{3}{*}{$\begin{array}{l}p \text { value (Kruskal- } \\
\text { Wallis test) }\end{array}$} \\
\hline & Sp. eosinophils $\geq 3 \%$ & Sp. eosinophils <3 \% & Sp. eosinophils $\geq 3 \%$ & Sp. eosinophils <3 \% & & \\
\hline & Sp. neutrophils $<76 \%$ & Sp. neutrophils $\geq 76 \%$ & Sp. neutrophils $\geq 76 \%$ & Sp. neutrophils $<76 \%$ & & \\
\hline $\begin{array}{l}\text { Total non-squamous cell count } \\
\left(\times 10^{6} / \mathrm{g}\right)\end{array}$ & $1.18(0.56-2.85)^{\ddagger^{*}}$ & $1.84(0.82-5.52)^{\ddagger^{*} \dagger}$ & $4.10(1.07-12.00)^{F^{*+}}$ & $0.81(0.40-2.07)$ & $0.64(0.37-1.30)$ & 0.0001 \\
\hline Viability (\%) & $64(50-75)$ & $79(68-88)^{\neq^{*+}}$ & $81(67-86)^{\ddagger^{*+}}$ & $64(48-75)$ & $67(53-78)$ & 0.0001 \\
\hline Squamous cells (\%) & $13(5-25)^{\ddagger^{*}}$ & $12(3-28)^{\ddagger^{*}}$ & $6(2-23)^{\ddagger^{*}}$ & $18(8-35)$ & $18(10-31)$ & 0.0001 \\
\hline \multicolumn{7}{|l|}{ Eosinophils } \\
\hline$\times 10^{3} / \mathrm{g}$ & $201(64-636)^{\ddagger^{*}}$ & $3(0-27)^{\neq+\S}$ & $271(46-685)^{\ddagger *}$ & $3(0-10)^{\ddagger}$ & $0(0-2)$ & 0.0001 \\
\hline$\%$ of non-squamous cells & $17.1(7.4-38.0)^{\neq^{*}}$ & $0.2(0.0-1.0)^{\ddagger \uparrow \S}$ & $5.2(3.4-8.8)^{f^{* *}+}$ & $0.4(0.0-1.0)^{\ddagger}$ & $0.0(0.0-0.4)$ & - \\
\hline \multicolumn{7}{|l|}{ Neutrophils } \\
\hline$\times 10^{3} / \mathrm{g}$ & $335(135-823)^{\ddagger}$ & $1504(707-4728)^{\ddagger^{*+}}$ & $3272(881-10992)^{\neq^{*} \dagger}$ & $270(90-795)$ & $210(63-563)$ & 0.0001 \\
\hline$\%$ of non-squamous cells & $33(16-50)^{*}$ & $87(82-93)^{\#^{*+}}$ & $83(79-88)^{*^{*+}}$ & $42(21-58)$ & $36(13-63)$ & - \\
\hline \multicolumn{7}{|l|}{ Macrophages } \\
\hline$\times 10^{3} / \mathrm{g}$ & $288(144-648)$ & $169(52-425)^{f^{* *}+}$ & $218(77-607)$ & 337 (167-853) & $285(120-568)$ & 0.0001 \\
\hline$\%$ of non-squamous cells & $27(16-43)^{\ddagger^{*}}$ & $9(4-13)^{f^{*}+}$ & $6(3-9)^{f^{*}+}$ & $46(29-61)$ & $46(30-67)$ & 0.0001 \\
\hline \multicolumn{7}{|l|}{ Lymphocytes } \\
\hline$\times 10^{3} / \mathrm{g}$ & $19(5-52)^{\ddagger^{*}}$ & $12(2-41)$ & $19(0-88)$ & $10(3-31)$ & $10(4-26)$ & 0.0012 \\
\hline$\%$ of non-squamous cells & $1.7(0.6-3.2)$ & $1.0(0.3-1.6)^{F^{*}+}$ & $0.6(0.0-1.8)^{\ddagger \dagger}$ & $1.4(0.5-2.8)$ & $1.8(0.8-3.4)$ & 0.0001 \\
\hline \multicolumn{7}{|l|}{ Epithelial cells } \\
\hline$\times 10^{3} / \mathrm{g}$ & $56(20-140)^{\ddagger}$ & $18(3-67)^{*^{*+}}$ & $15(0-106)^{*+}$ & $50(23-115)^{\ddagger}$ & $35(12-86)$ & 0.0001 \\
\hline$\%$ of non-squamous cells & $5.0(2.0-8.6)^{*}$ & $1.0(0.4-2.0)^{F^{*}+}$ & $0.6(0.2-1.8)^{F^{*}+}$ & $6.0(2.8-13.0)$ & $4.8(2.2-11.8)$ & 0.0001 \\
\hline
\end{tabular}

${ }^{\ddagger} p<0.005$, comparison with healthy subjects

$p<0.005$, comparison with paucigranulocytic asthma

${ }^{+} p<0.005$, comparison with eosinophilic asthma

${ }^{s} p<0.005$, comparison with mixed granulocytic asthma

Abbreviation: Sp. sputum 

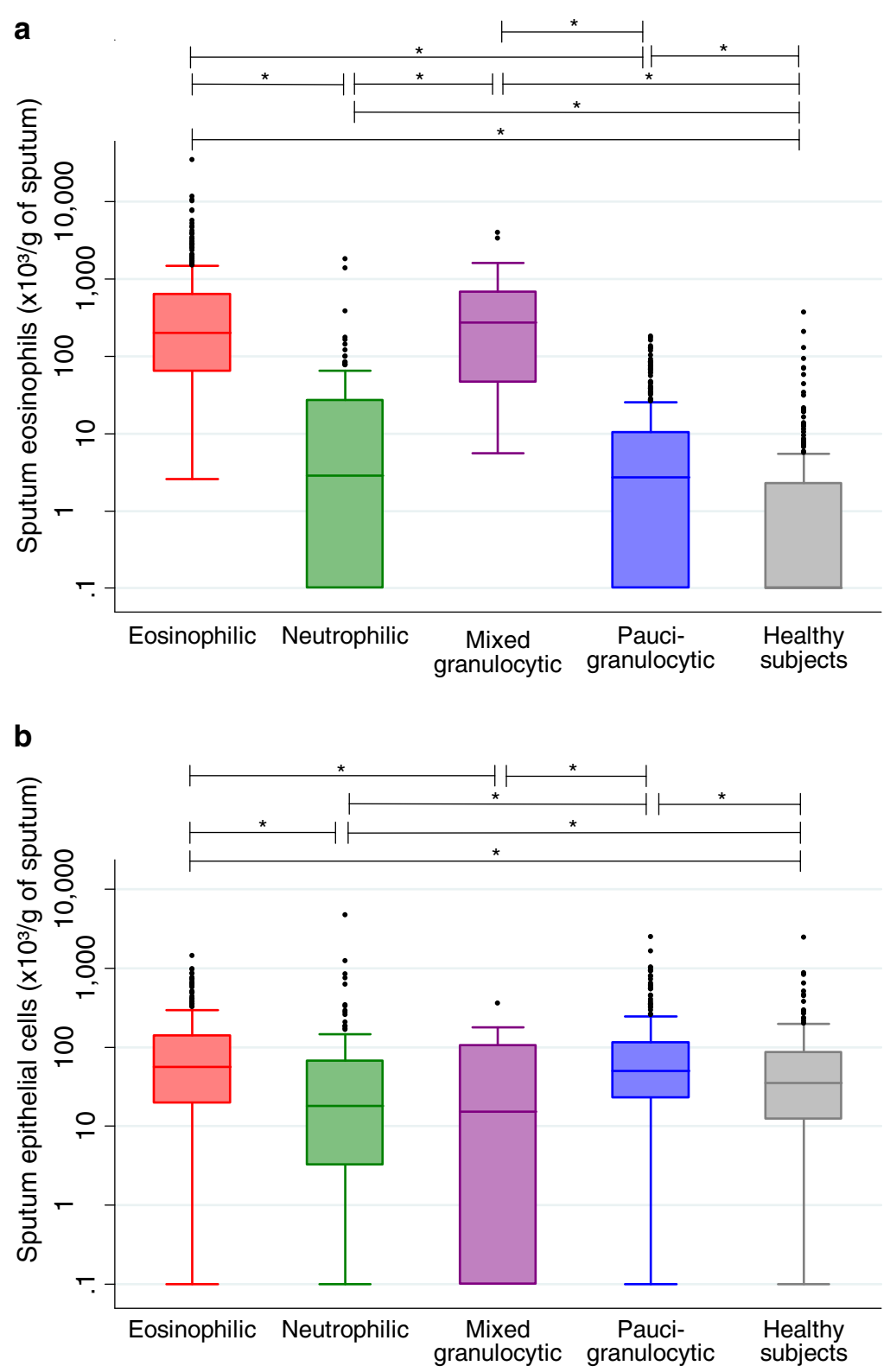

Fig. 1 Absolute sputum eosinophils (a) and absolute sputum epithelial cells (b) in asthma phenotypes and healthy subjects. ${ }^{*} p<0.005$. Values of 0 were assigned to 0.1 because of the use of a logarithmic scale

\section{Blood leukocyte counts and systemic inflammatory markers}

The blood leukocyte counts and systemic inflammatory markers of asthma phenotypes and healthy subjects are shown in Table 3. All subgroups of asthmatics showed an increased level of blood leukocytes compared with healthy subjects (Table 3 and Fig. 2a). As expected, eosinophilic and mixed granulocytic asthmatics had higher blood eosinophils (in absolute values and percentages) than neutrophilic, paucigranulocytic asthma and healthy subjects, but paucigranulocytic asthmatics also showed higher levels of absolute blood eosinophils than healthy subjects. Neutrophilic asthmatics had the highest level of blood neutrophils, compared to eosinophilic, paucigranulocytic asthma and healthy subjects, but both eosinophilic and paucigranulocytic asthmatics also had greater absolute blood neutrophils than healthy subjects. Absolute lymphocyte counts were mainly increased in eosinophilic and paucigranulocytic asthma. Only the group of eosinophilic asthma had increased absolute blood basophil counts compared to healthy subjects. Neutrophilic asthma was characterized by raised CRP and fibrinogen levels, while eosinophilic asthma had raised fibrinogen only, compared with healthy subjects (Table 3 and Fig. 2b). 
Table 3 Blood leukocyte counts and systemic inflammatory markers of asthmatics classified by phenotypes and healthy subjects

\begin{tabular}{|c|c|c|c|c|c|c|}
\hline & Eosinophilic asthma & Neutrophilic asthma & Mixed granulocytic asthma & Paucigranulocytic asthma & Healthy subjects ${ }^{a}$ & $p$ value (Kruskal- \\
\hline & Sp. eosinophils $\geq 3 \%$ & Sp. eosinophils $<3 \%$ & Sp. eosinophils $\geq 3 \%$ & Sp. eosinophils $<3 \%$ & & \\
\hline & Sp. neutrophils $<76 \%$ & Sp. neutrophils $\geq 76 \%$ & Sp. neutrophils $\geq 76 \%$ & Sp. neutrophils $<76 \%$ & & \\
\hline Blood leukocytes (x 103/ML) & $7.76(6.52-8.87)^{\ddagger}$ & $7.75(6.43-10.00)^{\ddagger}$ & $8.02(6.16-10.83)^{\ddagger}$ & $7.25(6.02-8.75)^{\ddagger}$ & $6.12(5.03-7.37)$ & 0.0001 \\
\hline \multicolumn{7}{|l|}{ Blood eosinophils } \\
\hline$/ \mu \mathrm{L}$ & $315(202-513)^{\ddagger^{*}}$ & $129(79-228)^{+\S}$ & $289(216-449)^{\ddagger^{*}}$ & $140(85-227)^{\ddagger}$ & $106(68-172)$ & 0.0001 \\
\hline$\%$ & $4.2(2.8-6.2)^{\ddagger^{*}}$ & $1.7(1.0-3.0)^{\dagger \S}$ & $3.9(2.7-5.8)^{\mp^{*}}$ & $2.0(1.1-3.0)$ & $1.8(1.0-3.0)$ & 0.0001 \\
\hline \multicolumn{7}{|l|}{ Blood neutrophils } \\
\hline$/ \mu \mathrm{L}$ & $4045(3204-5140)^{\ddagger}$ & $4847(3260-6323)^{\neq^{*+}}$ & 4207 (2834-6610) & $3971(3156-5280)^{\ddagger}$ & 3416 (2610-4188) & 0.0001 \\
\hline$\%$ & $54.0(47.6-60.1)^{*}$ & $59.5(52.5-69.3)^{*}+$ & $57.6(44.4-62.4)$ & $56.5(50.3-62.2)$ & $54.4(49.6-62.1)$ & 0.0001 \\
\hline \multicolumn{7}{|l|}{ Blood monocytes } \\
\hline$/ \mu \mathrm{L}$ & $515(396-667)^{\ddagger^{*}}$ & $492(408-656)^{\ddagger}$ & $564(448-775)^{\ddagger}$ & $461(362-608)$ & $399(330-550)$ & 0.0001 \\
\hline$\%$ & $6.7(5.1-8.3)$ & $6.5(5.4-8.2)$ & $7.2(5.6-9.0)$ & $6.4(5.1-8.2)$ & $7.1(5.7-8.2)$ & $>0.05$ \\
\hline \multicolumn{7}{|l|}{ Blood lymphocytes } \\
\hline$/ \mu \mathrm{L}$ & $2358(1918-2809)^{\ddagger}$ & $2249(1765-2807)$ & $2180(1887-2869)$ & $2337(1894-2904)^{\ddagger}$ & $2009(1611-2386)$ & 0.0001 \\
\hline$\%$ & $31.8(26.0-38.3)$ & $29.4(22.5-37.1)^{\ddagger^{*}}$ & $29.1(21.0-38.2)$ & $32.7(27.3-38.2)$ & $33.9(26.5-37.7)$ & 0.006 \\
\hline \multicolumn{7}{|l|}{ Blood basophils } \\
\hline$/ \mu \mathrm{L}$ & $42(29-63)^{\ddagger^{*}}$ & $35(23-51)^{\dagger}$ & $39(27-55)$ & $34(24-52)$ & $34(24-46)$ & 0.0001 \\
\hline$\%$ & $0.6(0.4-0.8)^{*}$ & $0.5(0.3-0.6)^{\ddagger \dagger}$ & $0.5(0.4-0.7)$ & $0.5(0.3-0.7)$ & $0.5(0.4-0.8)$ & 0.0001 \\
\hline $\operatorname{CRP}(\mathrm{mg} / \mathrm{L})^{\mathrm{b}}$ & $1.4(0.6-3.6)$ & $1.9(0.8-5.3)^{\ddagger}$ & $2.9(0.6-8.9)$ & $1.6(0.6-4.5)$ & $1.1(0.4-2.4)$ & 0.034 \\
\hline Fibrinogen $(\mathrm{g} / \mathrm{L})^{c}$ & $3.2(2.8-3.8)^{\ddagger}$ & $3.2(2.8-3.7)^{\ddagger}$ & $3.3(2.7-3.9)$ & $3.1(2.7-3.6)$ & $2.9(2.6-3.2)$ & 0.0008 \\
\hline
\end{tabular}

${ }^{\mathrm{a}}$ Data available for 96 healthy subjects

${ }^{\mathrm{b} D a t a}$ available for 208 eosinophilic, 92 neutrophilic, 23 mixed granulocytic, 186 paucigranulocytic and 63 healthy subjects 'Data available for 194 eosinophilic, 85 neutrophilic, 21 mixed granulocytic, 174 paucigranulocytic and 59 healthy subjects ${ }^{\ddagger} p<0.005$, comparison with healthy subjects

$p<0.005$, comparison with paucigranulocytic asthma

${ }^{+} p<0.005$, comparison with eosinophilic asthma

${ }^{s} p<0.005$, comparison with mixed granulocytic asthma

Abbreviations: CRP C-reactive protein; Sp. sputum 

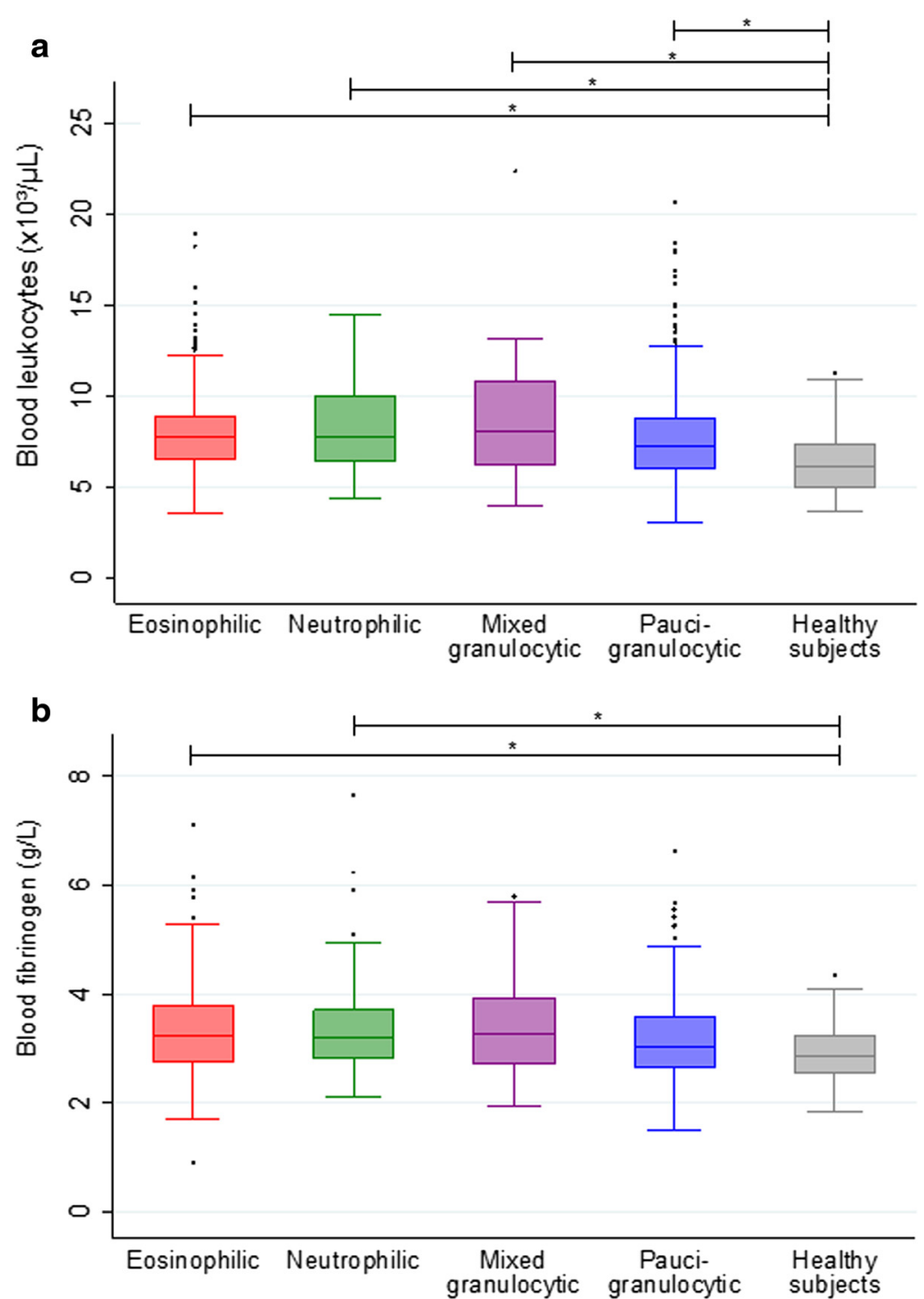

Fig. 2 Blood leukocytes (a) and blood fibrinogen (b) in asthma phenotypes and healthy subjects. ${ }^{*} p<0.005$

\section{Analysis of asthma phenotypes according to treatment with inhaled corticosteroids (ICS) and comparison with healthy subjects}

For each phenotype, we compared sputum and blood cell counts between patients treated and not treated with ICS and we also compared these subgroups with healthy subjects (Table 4 and Additional file 1: Table S1). In steroidnaïve paucigranulocytic patients, as in the whole group of paucigranulocytic asthma, there was an increase in sputum eosinophil counts, compared to healthy subjects. With respect to systemic inflammation, blood leukocyte, eosinophil and lymphocyte counts were increased in steroid-naïve paucigranulocytic asthmatics, compared to healthy subjects $(p<0.0042$ : level of statistical significance after Bonferroni correction, Table 4). Paucigranulocytic asthmatics receiving inhaled corticosteroids showed an increase in sputum eosinophils and epithelial cells, and an increase in blood leukocyte, neutrophil and lymphocyte numbers $(p<0.0042$, Table 4). We did not find any statistically significant difference between patients treated and not treated with ICS in the subgroups of paucigranulocytic, neutrophilic and mixed granulocytic asthma $(p>0.0042$, Table 4 and Additional file 1: Table S1). In eosinophilic asthmatics, patients treated with ICS presented higher levels of sputum epithelial cells, blood leukocytes and blood neutrophils, as compared with eosinophilic patients not treated with ICS $(p<0.0042$, Additional file 1: Table S1).

\section{Analysis of asthma phenotypes classified with a threshold of sputum eosinophils of $1.01 \%$}

We also used $1.01 \%$ as the threshold of sputum eosinophils to define asthma phenotypes. When using this cutoff value, 
Table 4 Sputum and blood cell counts of non-eosinophilic phenotypes classified by ICS treatment and healthy subjects

\begin{tabular}{|c|c|c|c|c|c|c|c|}
\hline & $\begin{array}{l}\text { Paucigranulocytic asthma, } \\
\text { not treated with ICS }\end{array}$ & $\begin{array}{l}\text { Paucigranulocytic asthma, } \\
\text { treated with ICS }\end{array}$ & ICS- vs ICS+ & $\begin{array}{l}\text { Neutrophilic asthma, } \\
\text { not treated with ICS }\end{array}$ & $\begin{array}{l}\text { Neutrophilic asthma, } \\
\text { treated with ICS }\end{array}$ & ICS- vs ICS+ & Healthy subjects \\
\hline N & 123 & 195 & - & 47 & 87 & - & 194 \\
\hline $\begin{array}{l}\text { Sputum total non-squamous cell } \\
\text { count }\left(\times 10^{6} / \mathrm{g}\right)\end{array}$ & $0.93(0.43-2.12)$ & $0.69(0.36-1.61)$ & NS & $1.45(0.82-4.65)^{\ddagger}$ & $2.10(0.75-8.02)^{\ddagger}$ & NS & $0.64(0.37-1.30)$ \\
\hline Sputum viability (\%) & $65(48-78)$ & $64(48-75)$ & NS & $78(68-87)^{\ddagger}$ & $80(68-88)^{\ddagger}$ & NS & $67(53-78)$ \\
\hline Sputum squamous cells (\%) & $16(7-30)$ & $19(10-37)$ & NS & $18(5-30)$ & $7(2-27)^{\ddagger}$ & NS & $18(10-31)$ \\
\hline Sputum eosinophils $\left(\times 10^{3} / \mathrm{g}\right)$ & $4(0-15)^{\ddagger}$ & $2(0-10)^{\ddagger}$ & NS & $2(0-28)^{\ddagger}$ & $4(0-27)^{\ddagger}$ & NS & $0(0-2)$ \\
\hline Sputum neutrophils $\left(\times 10^{3} / \mathrm{g}\right)$ & $315(97-994)$ & $245(86-710)$ & NS & $1197(707-3776)^{\ddagger}$ & $1879(593-7060)^{\ddagger}$ & NS & $210(63-563)$ \\
\hline Sputum macrophages (x 103/g) & $451(188-864)^{\ddagger}$ & $280(154-853)$ & NS & $127(33-396)^{\ddagger}$ & $182(52-436)$ & NS & $285(120-568)$ \\
\hline Sputum lymphocytes (x 103/g) & $12(3-45)$ & $10(2-24)$ & NS & 15 (4-41) & $11(1-39)$ & NS & $10(4-26)$ \\
\hline Sputum epithelial cells (x 103/g) & $48(19-113)$ & $52(25-120)^{\ddagger}$ & NS & $19(4-44)$ & $18(3-73)$ & NS & $35(12-86)$ \\
\hline Blood leukocytes $\left(\times 10^{3} / \mu \mathrm{L}\right)^{\mathrm{a}}$ & $6.87(5.85-8.51)^{\ddagger}$ & $7.47(6.32-9.06)^{\ddagger}$ & NS & $7.87(6.41-9.93)^{\ddagger}$ & $7.71(6.44-10.06)^{\ddagger}$ & NS & $6.12(5.03-7.37)$ \\
\hline Blood eosinophils $(/ \mu L)^{a}$ & $145(101-217)^{\ddagger}$ & $139(74-229)$ & NS & $172(63-293)$ & $123(80-215)$ & NS & $106(68-172)$ \\
\hline Blood neutrophils $(/ \mu L)^{a}$ & 3598 (2924-5119) & $4211(3392-5392)^{\ddagger}$ & NS & $4804(3158-6021)^{\ddagger}$ & $4885(3403-6969)^{\ddagger}$ & NS & 3416 (2610-4188) \\
\hline Blood monocytes $(/ \mu L)^{a}$ & $461(353-592)$ & $463(370-615)^{\ddagger}$ & NS & $476(387-648)$ & $519(419-669)^{\ddagger}$ & NS & $399(330-550)$ \\
\hline Blood lymphocytes $(/ \mu \mathrm{L})^{a}$ & $2352(1891-2967)^{\ddagger}$ & $2321(1900-2855)^{\ddagger}$ & NS & $2518(1989-3035)^{\ddagger}$ & $2167(1675-2726)$ & NS & 2009 (1611-2386) \\
\hline Blood basophils $(/ \mu \mathrm{L})^{\mathrm{a}}$ & $35(25-52)$ & $33(24-51)$ & NS & 40 (29-53) & $33(22-50)$ & NS & $34(24-46)$ \\
\hline
\end{tabular}

\section{${ }^{a}$ Data available for 96 healthy subjects}

${ }^{\ddagger} p<0.0042$, comparison with healthy subjects

Abbreviation: ICS inhaled corticosteroid 
there was no statistically significant difference in sputum and blood eosinophils (in absolute values and percentages) between neutrophilic or paucigranulocytic asthma, and healthy subjects (the level of statistical significance after Bonferroni correction being a $p$ value $<0.005$, Table 5). However, there was a strong trend for greater absolute sputum eosinophil count in paucigranulocytic asthmatics versus healthy subjects $(p=0.005)$. In the paucigranulocytic asthma subgroup defined with this cutoff value, absolute sputum epithelial cells $\left(50(24-115) \times 10^{3} / \mathrm{g}\right)$, blood leukocytes $\left(7.21(5.82-8.66) \times 10^{3} / \mu \mathrm{L}\right)$, absolute blood neutrophils (3900 (3083-5299)/ $\mathrm{L})$ and absolute blood lymphocytes $(2334(1891-2904) / \mu \mathrm{L})$ were still greater than in healthy subjects $(p<0.005)$.

\section{Analysis according to atopy}

The blood and sputum eosinophil counts of asthmatics and healthy subjects according to their atopic status are presented in Table 6. We did not observe any statistically significant difference in these cells between atopic and non-atopic healthy subjects or asthmatics (the level of statistical significance after Bonferroni correction being a $p$ value $<0.0083$ ).

\section{Analysis according to smoking habits}

The proportion of asthma inflammatory phenotypes was analyzed according to the smoking status of patients (Fig. 3), and was not different between non-smokers, exsmokers and current smokers $(p=0.6)$. There was however a correlation between the number of pack-years and the percentage of sputum neutrophils but this correlation was absent with sputum eosinophils (Fig. 4).

\section{Discussion}

Our study shows that the four inflammatory asthma phenotypes display raised sputum eosinophilia compared to healthy subjects. Eosinophilic asthma showed higher levels of absolute sputum neutrophils and lymphocytes than healthy subjects, while neutrophilic asthmatics had a reduced number of sputum macrophages and epithelial cells. In addition, all phenotypes had increased systemic inflammation reflected by raised total circulating leukocyte counts, mainly accounted for by raised granulocytic blood cell counts compared to healthy subjects.

As previously shown [2], our data indicate that neutrophilic and mixed granulocytic inflammation is more intense than pure eosinophilic and paucigranulocytic ones as the total number of cells per gram of sputum was clearly increased in these two first forms of groups. This might be related to the larger pool of circulating neutrophils and the variety of stimuli able to recruit neutrophils into the airways [15]. Our results showing greater viability in neutrophilic and mixed granulocytic asthma are also in keeping with those reported by Simpson et al. [2]. It may seem paradoxical as neutrophils are recognized to be more fragile than eosinophils in cell culture [16], but this may suggest greater cell turnover and short time of residency of neutrophils in the airways [17].

It has been suggested that non-eosinophilic asthmatics are poorly responsive to inhaled corticosteroids $[8,18]$. One interesting finding of our study is the fact that the so called "non-eosinophilic phenotypes" (neutrophilic and paucigranulocytic phenotypes) actually had more sputum eosinophils than healthy subjects, pointing to a low grade

Table 5 Eosinophilic inflammation of asthmatics classified by phenotypes (with a sputum eosinophil cutoff of $1.01 \%$ ) and healthy subjects

\begin{tabular}{|c|c|c|c|c|c|c|}
\hline & Eosinophilic asthma & Neutrophilic asthma & $\begin{array}{l}\text { Mixed granulocytic } \\
\text { asthma }\end{array}$ & $\begin{array}{l}\text { Paucigranulocytic } \\
\text { asthma }\end{array}$ & \multirow[t]{3}{*}{$\begin{array}{l}\text { Healthy } \\
\text { subjects }\end{array}$} & \multirow{3}{*}{$\begin{array}{l}\text { p value } \\
\text { (Kruskal- } \\
\text { Wallis test) }\end{array}$} \\
\hline & Sp. eosinophils $\geq 1.01 \%$ & Sp. eosinophils <1.01 \% & Sp. eosinophils $\geq 1.01 \%$ & Sp. eosinophils $<1.01 \%$ & & \\
\hline & Sp. neutrophils <76 \% & Sp. neutrophils $\geq 76 \%$ & Sp. neutrophils $\geq 76 \%$ & Sp. neutrophils <76 \% & & \\
\hline $\begin{array}{l}\mathrm{N} \text { (\% of } \\
\text { asthmatics) }\end{array}$ & $429(51)$ & $106(13)$ & $59(7)$ & $239(29)$ & 194 & - \\
\hline \multicolumn{7}{|l|}{$\begin{array}{l}\text { Sputum } \\
\text { eosinophils }\end{array}$} \\
\hline$\times 10^{3} / \mathrm{g}$ & $127(38-471)^{\ddagger^{*}}$ & $0(0-9)^{\dagger \S}$ & $77(31-380)^{\ddagger^{*}}$ & $0(0-5)^{\ddagger \ddagger}$ & $0(0-2)$ & 0.0001 \\
\hline $\begin{array}{l}\% \text { of non- } \\
\text { squamous cells }\end{array}$ & $11.8(4.0-30.8)^{\ddagger^{*}}$ & $0.0(0.0-0.5)^{\dagger \S}$ & $3.0(1.8-6.0)^{f^{*+}}$ & $0.2(0.0-0.5)$ & $0.0(0.0-0.4)$ & 0.0001 \\
\hline \multicolumn{7}{|l|}{$\begin{array}{l}\text { Blood } \\
\text { eosinophils }\end{array}$} \\
\hline$/ \mu \mathrm{L}$ & $289(181-444)^{\ddagger^{*}}$ & $123(62-220)^{\dagger \S}$ & $266(126-430)^{\ddagger^{*}}$ & $133(78-200)$ & $106(68-172)$ & 0.0001 \\
\hline$\%$ & $3.8(2.4-5.6)^{\ddagger^{*}}$ & $1.7(0.9-3.0)^{\dagger \S}$ & $2.9(1.8-5.6)^{\ddagger^{*}}$ & $1.9(1.0-2.7)$ & $1.8(1.0-3.0)$ & 0.0001 \\
\hline
\end{tabular}

${ }^{a}$ Data available for 96 healthy subjects

${ }^{\ddagger} p<0.005$, comparison with healthy subjects

${ }^{\ddagger \ddagger} p=0.005$, comparison with healthy subjects

* $p<0.005$, comparison with paucigranulocytic asthma

${ }^{+} p<0.005$, comparison with eosinophilic asthma

${ }^{s} p<0.005$, comparison with mixed granulocytic asthma

Abbreviation: Sp. sputum 
Table 6 Eosinophilic inflammation of asthmatics and healthy subjects according to atopy

\begin{tabular}{|c|c|c|c|c|c|}
\hline & Non-atopic asthmatics & Atopic asthmatics & Non-atopic healthy subjects & Atopic healthy subjects & $\begin{array}{l}\text { p value (Kruskal-Wallis } \\
\text { test) }\end{array}$ \\
\hline N & 344 & 464 & 118 & 46 & - \\
\hline \multicolumn{6}{|l|}{ Sputum eosinophils } \\
\hline$\times 10^{3} / \mathrm{g}$ & $19(1-173)^{\ddagger^{*}}$ & $34(3-181)^{\ddagger^{*}}$ & $0(0-3)$ & $0(0-4)$ & 0.0001 \\
\hline$\%$ of non-squamous cells & $1.6(0.2-9.6)^{f^{*}}$ & $2.8(0.2-13.8)^{\ddagger^{*}}$ & $0.0(0.0-0.4)$ & $0.0(0.0-0.5)$ & 0.0001 \\
\hline \multicolumn{6}{|l|}{ Blood eosinophils ${ }^{a}$} \\
\hline$/ \mu \mathrm{L}$ & $188(109-327)^{\ddagger}$ & $217(124-375)^{\ddagger^{*}}$ & $93(62-135)$ & $134(80-202)$ & 0.0001 \\
\hline$\%$ & $2.6(1.4-4.0)^{\ddagger}$ & $3.0(1.7-4.7)^{\ddagger}$ & $1.5(0.9-2.9)$ & $2.1(1.6-3.0)$ & 0.0001 \\
\hline
\end{tabular}

${ }^{a}$ Data available for 54 non-atopic healthy subjects and 31 atopic healthy subjects

${ }^{\ddagger} p<0.0083$, comparison with non-atopic healthy subjects

* $p<0.0083$, comparison with atopic healthy subjects

eosinophilic inflammation in these phenotypes. This could explain the fact that some patients with non-eosinophilic asthma responded to inhaled corticosteroids in the study of Cowan et al. [19], even though a limitation of this study was the absence of placebo-controlled design. Also of interest is the fact that eosinophilic asthmatics had more absolute sputum neutrophils compared to healthy subjects. It is likely to reflect that pathways leading to the recruitment of eosinophils and neutrophils in the airways may co-exist or act through a same effector in asthma [20].

It is noteworthy that absolute sputum lymphocyte counts were increased when there was significant eosinophilic infiltration. Although little attention has been paid to lymphocytes in sputum so far because of their small number (representing approximately 1-2 \%), our finding is in keeping with the role of lymphocytes in organizing eosinophilic inflammation, a concept which has been established by bronchial biopsy and bronchoalveolar lavage analyses [21, 22].

A particularly low absolute number of macrophages was observed in neutrophilic asthma. Further to this decreased amount of macrophages in the airways, Simpson et al. [23] described an impaired capacity of sputum macrophages to phagocytose apoptotic material in non-eosinophilic asthma, which may explain chronic inflammation and accumulation of airway neutrophils.
In keeping with epithelial alteration in asthma [24], we found an increased epithelial cell number in sputum from eosinophilic and paucigranulocytic asthma. By contrast, epithelial cell count was particularly low in the groups displaying intense neutrophilic inflammation. The presence of epithelial cells in sputum samples may reflect epithelial desquamation. Therefore, it is somewhat surprising to find lower epithelial cell counts in neutrophilic asthma. Our clinical feeling is that the cough effort during sputum induction may favor epithelial shedding and our experience is that effort to produce sputum is less when patients have intense neutrophilic inflammation, which may prevent epithelial cell desquamation.

In contrast to COPD [25], little attention has been paid to systemic inflammation in asthma, so far. Our data show striking elevation of total blood leukocyte counts in all asthmatic phenotypes compared to healthy subjects. There was however no difference between asthma phenotypes in this respect, which is in keeping with the recent study of Zhang et al. [11]. Although raised circulating leukocytes might have been expected in patients with eosinophilic, neutrophilic and mixed granulocytic asthma, this finding was somewhat more surprising in paucigranulocytic asthma.

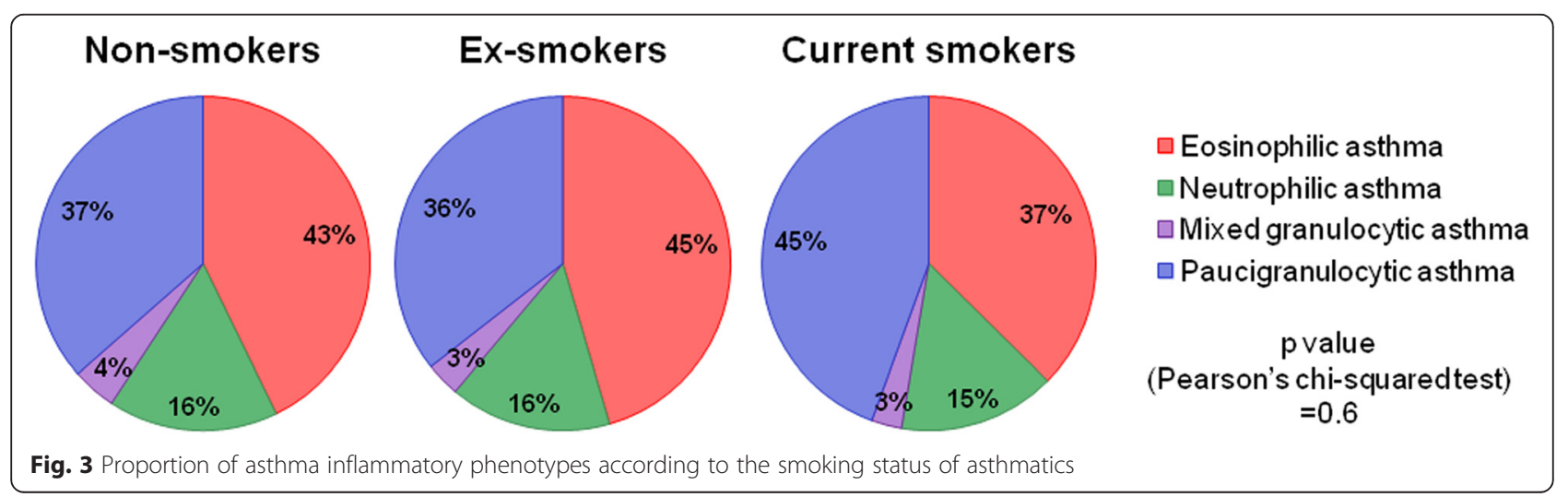



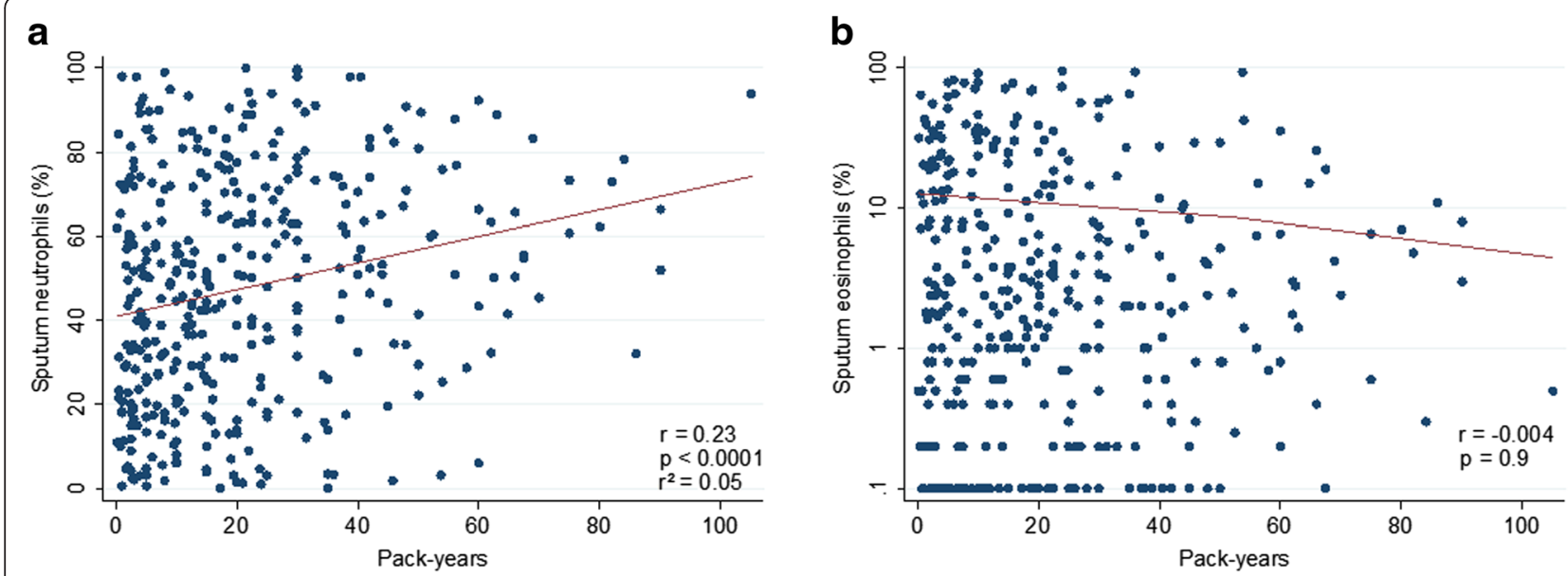

Fig. 4 Relationship between sputum neutrophils (a) and eosinophils (b) and the number of pack-years in ex- and current smoker asthmatics. For sputum eosinophils, values of 0 were assigned to 0.1 because of the use of a logarithmic scale

As previously shown $[3,11]$, patients with a raised sputum eosinophilic inflammation also had the highest levels of blood eosinophils (absolute values or percentages). Moreover, we found that the highest levels of absolute blood neutrophils were observed in neutrophilic and mixed granulocytic asthma, although the results did not reach statistical significance in this last phenotype, probably due to the small number of patients. Interestingly, paucigranulocytic asthmatics also had raised absolute circulating eosinophils and neutrophils compared to healthy subjects. Overall it points to a granulocytic inflammation in all asthma phenotypes.

Like in sputum, absolute lymphocyte counts were increased in blood of patients with eosinophilic asthma. Interestingly, levels of absolute blood basophils were also higher in these patients, which is consistent with a previous report showing a concomitant increase in circulating eosinophils and basophils after allergen inhalation in atopic asthmatics [26].

Supporting the concept of low grade systemic inflammation in asthma, we found a mild but significant increase in fibrinogen levels in eosinophilic and neutrophilic asthma, while increase in CRP was essentially observed in asthmatics with intense airway neutrophilic infiltration, as previously shown [27].

The group of paucigranulocytic asthmatics may be considered heterogeneous as almost two third of the patients were receiving ICS, which may have attenuated an airway inflammation initially present before the start of the treatment. Therefore, it is interesting to notice that mild airway and systemic eosinophilic inflammation, together with raised circulating lymphocyte number, were still present in those patients not treated with ICS. One limitation of our study is the retrospective design that precludes any further phenotypic characterization of circulating lymphocytes.
Several interventional studies have shown that ICS therapy decreases sputum eosinophils [28, 29] and increases sputum neutrophils [19]. In our study, we did not find any statistically significant difference in these sputum cells between patients treated and not treated with ICS. However, because of its cross-sectional design, our study does not question the impact of ICS on eosinophil and neutrophil counts. We believe that the persistence of significant sputum eosinophilia in ICS treated asthmatics reflects the severity of the inflammatory process in these patients.

Paucigranulocytic asthma is defined as a sputum eosinophil count $<3 \%$ in our study. Others have proposed a cutoff at $1.01 \%$ [2]. When choosing this cutoff in our patients, we increase the proportion of eosinophilic asthma from 42 to $51 \%$ and we correspondingly decrease the proportion of paucigranulocytic asthma from 38 to $29 \%$. With this cutoff at $1.01 \%$, the group of paucigranulocytic asthmatics still displayed signs of airway and systemic inflammation, although they did not show increased circulating blood eosinophils anymore. It is interesting to postulate that the paucigranulocytic phenotype may be composed of some asthmatics presenting low grade inflammation and others without any sign of airway and systemic inflammation.

Some studies have shown that the eosinophilic inflammation is somewhat related to atopy [3, 30]. Our data suggest that the eosinophilic trait observed in all asthma phenotypes is independent of the atopic status with non-atopic asthmatics not displaying statistically different sputum and blood eosinophil counts from their atopic counterparts.

There was no significant change in the proportion of inflammatory phenotypes according to the smoking status. However, in contrast to sputum eosinophils, sputum 
neutrophil count appears to be partly associated with the cumulative smoking history expressed as pack-years. Previous studies failed to find this relationship [31, 32] but they were performed on a limited number of patients. This association remains however rather limited based on our results, where pack-years can only account for $5 \%$ of the neutrophil variability.

It is known that asthma inflammatory phenotypes may somewhat vary over time [2,33]. One limitation of our study is its cross-sectional design with sputum analyzed at only one time point.

\section{Conclusions}

Overall, this study demonstrates that a significant eosinophilic inflammation is present across all categories of asthma, and that paucigranulocytic asthma may be seen as a low grade inflammatory disease. Whether steroid-naïve or a part of steroid-treated paucigranulocytic asthmatics may do without ICS should be investigated in long term trials, but our data incite to be cautious in clinical practice for now.

\section{Ethics approval and consent to participate}

This retrospective study was conducted with the approval from the ethics committee of the University Hospital of Liege (Reference 2015/193). Informed consents were obtained from healthy subjects. As for asthmatic patients, all procedures were performed in the context of clinical practice and the retrospective data collection was conducted with the approval from the abovementioned ethics committee.

\section{Consent for publication}

Not applicable.

\section{Availability of data and material}

The data supporting our results are presented within the article (and its Additional file 1: Table S1).

\section{Additional file}

Additional file 1: Table S1. Sputum and blood cell counts of eosinophilic phenotypes classified by ICS treatment and healthy subjects. (DOCX $29.3 \mathrm{~kb}$ )

\section{Abbreviations}

BMl: body mass index; CRP: C-reactive protein; FENO: fractional exhaled nitric oxide; FEV : forced expiratory volume in 1 second; FVC: forced vital capacity; ICS: inhaled corticosteroid; LABA: long-acting $\beta 2$-agonist; LTRA: leukotriene receptor antagonist; OCS: oral corticosteroid; PC20M: provocative concentration of methacholine causing a $20 \%$ fall in $\mathrm{FEV}_{1}$.

\section{Competing interests}

The authors declare that they have no competing interests.

\section{Authors' contributions}

SD designed the study, helped to collect the data, analyzed and interpreted the data and drafted the manuscript. FS collected the data, helped to draft the manuscript and revised it critically for important intellectual content. $\mathrm{MH}$ and VP collected the data and revised the manuscript critically for important intellectual content. TVH helped to analyze the data and revised the manuscript critically for important intellectual content. RL designed the study, collected the data, interpreted the data and drafted the manuscript. All authors read and approved the final manuscript.

\section{Acknowledgements}

We acknowledge the Fonds Léon Fredericq for funding training in statistics.

Funding

This work was supported by a federal grant IAP P7/30.

\section{Author details}

'Department of Respiratory Medicine, CHU Liege, Sart-Tilman B35, 4000 Liege, Belgium. ${ }^{2} \mathrm{GIGA}$ I $^{3}$ Research Group, University of Liege, Liege, Belgium. ${ }^{3}$ Department of Clinical and Hospital Pharmacy, University of Liege, Liege, Belgium.

Received: 10 November 2015 Accepted: 18 March 2016

Published online: 05 April 2016

References

1. Pavord ID, Pizzichini MM, Pizzichini E, Hargreave FE. The use of induced sputum to investigate airway inflammation. Thorax. 1997;52:498-501.

2. Simpson JL, Scott R, Boyle MJ, Gibson PG. Inflammatory subtypes in asthma: assessment and identification using induced sputum. Respirol Carlton Vic. 2006;11:54-61.

3. Schleich FN, Manise M, Sele J, Henket M, Seidel L, Louis R. Distribution of sputum cellular phenotype in a large asthma cohort: predicting factors for eosinophilic vs neutrophilic inflammation. BMC Pulm Med. 2013;13:11.

4. in't Veen JC, de Gouw HW, Smits HH, Sont JK, Hiemstra PS, Sterk PJ, et al. Repeatability of cellular and soluble markers of inflammation in induced sputum from patients with asthma. Eur Respir J. 1996;9:2441-7.

5. Bacci E, Cianchetti S, Carnevali S, Bartoli ML, Dente FL, Di Franco A, et al. Induced sputum is a reproducible method to assess airway inflammation in asthma. Mediators Inflamm. 2002;11:293-8.

6. Vlachos-Mayer H, Leigh R, Sharon RF, Hussack P, Hargreave FE. Success and safety of sputum induction in the clinical setting. Eur Respir J. 2000:16:997-1000

7. Green RH, Brightling CE, Woltmann G, Parker D, Wardlaw AJ, Pavord ID. Analysis of induced sputum in adults with asthma: identification of subgroup with isolated sputum neutrophilia and poor response to inhaled corticosteroids. Thorax. 2002;57:875-9.

8. Haldar P, Pavord ID. Noneosinophilic asthma: a distinct clinical and pathologic phenotype. J Allergy Clin Immunol. 2007;119:1043-52. quiz 1053-4.

9. Hastie AT, Moore WC, Meyers DA, Vestal PL, Li H, Peters SP, et al. Analyses of asthma severity phenotypes and inflammatory proteins in subjects stratified by sputum granulocytes. J Allergy Clin Immunol. 2010;125:1028-36. e13.

10. Porsbjerg C, Lund TK, Pedersen L, Backer V. Inflammatory subtypes in asthma are related to airway hyperresponsiveness to mannitol and exhaled NO. J Asthma Off J Assoc Care Asthma. 2009;46:606-12.

11. Zhang $X-Y$, Simpson $J$, Powell H, Yang IA, Upham JW, Reynolds PN, et al. Full blood count parameters for the detection of asthma inflammatory phenotypes. Clin Exp Allergy J Br Soc Allergy Clin Immunol. 2014;44:1137-45.

12. Delvaux $M$, Henket $M$, Lau L, Kange $P$, Bartsch $P$, Djukanovic $R$, et al. Nebulised salbutamol administered during sputum induction improves bronchoprotection in patients with asthma. Thorax. 2004;59:111-5.

13. Gershman NH, Wong HH, Liu JT, Mahlmeister MJ, Fahy JV. Comparison of two methods of collecting induced sputum in asthmatic subjects. Eur Respir J. 1996:9:2448-53.

14. Louis R, Godinas L, Schleich F. Induced Sputum - Towards Normal Values. Non Invasive Assessment of airways inflammation in asthma and COPD. Athens: Paschalidis Medical Publications; 2011. p. 113-23.

15. Douwes J, Gibson P, Pekkanen J, Pearce N. Non-eosinophilic asthma: importance and possible mechanisms. Thorax. 2002;57:643-8. 
16. Meagher LC, Cousin JM, Seckl JR, Haslett C. Opposing effects of glucocorticoids on the rate of apoptosis in neutrophilic and eosinophilic granulocytes. J Immunol Baltim Md 1950. 1996;156:4422-8.

17. Kamath AV, Pavord ID, Ruparelia PR, Chilvers ER. Is the neutrophil the key effector cell in severe asthma? Thorax. 2005;60:529-30.

18. Louis R, Schleich F, Barnes PJ. Corticosteroids: still at the frontline in asthma treatment? Clin Chest Med. 2012;33:531-41.

19. Cowan DC, Cowan JO, Palmay R, Williamson A, Taylor DR. Effects of steroid therapy on inflammatory cell subtypes in asthma. Thorax. 2010;65:384-90.

20. Cosmi L, Liotta F, Maggi E, Romagnani S, Annunziato F. Th17 cells: new players in asthma pathogenesis. Allergy. 2011;66:989-98.

21. Bradley BL, Azzawi M, Jacobson M, Assoufi B, Collins JV, Irani AM, et al. Eosinophils, T-lymphocytes, mast cells, neutrophils, and macrophages in bronchial biopsy specimens from atopic subjects with asthma: comparison with biopsy specimens from atopic subjects without asthma and normal control subjects and relationship to bronchial hyperresponsiveness. J Allergy Clin Immunol. 1991;88:661-74.

22. Walker C, Kaegi MK, Braun P, Blaser K. Activated T cells and eosinophilia in bronchoalveolar lavages from subjects with asthma correlated with disease severity. J Allergy Clin Immunol. 1991;88:935-42.

23. Simpson JL, Gibson PG, Yang IA, Upham J, James A, Reynolds PN, et al. Impaired macrophage phagocytosis in non-eosinophilic asthma. Clin Exp Allergy J Br Soc Allergy Clin Immunol. 2013;43:29-35.

24. Al-Muhsen S, Johnson JR, Hamid Q. Remodeling in asthma. J Allergy Clin Immunol. 2011;128:451-62. quiz 463-4.

25. Dickens JA, Miller BE, Edwards LD, Silverman EK, Lomas DA, Tal-Singer R, et al. COPD association and repeatability of blood biomarkers in the ECLIPSE cohort. Respir Res. 2011;12:146.

26. Gibson PG, Manning PJ, O'Byrne PM, Girgis-Gabardo A, Dolovich J, Denburg $J A$, et al. Allergen-induced asthmatic responses. Relationship between increases in airway responsiveness and increases in circulating eosinophils, basophils, and their progenitors. Am Rev Respir Dis. 1991;143:331-5.

27. Wood LG, Baines K, Fu J, Scott HA, Gibson PG. The neutrophilic inflammatory phenotype is associated with systemic inflammation in asthma. Chest. 2012;142:86-93.

28. Berry M, Morgan A, Shaw DE, Parker D, Green R, Brightling C, et al. Pathological features and inhaled corticosteroid response of eosinophilic and non-eosinophilic asthma. Thorax. 2007;62:1043-9.

29. Jatakanon A, Kharitonov S, Lim S, Barnes PJ. Effect of differing doses of inhaled budesonide on markers of airway inflammation in patients with mild asthma. Thorax. 1999;54:108-14.

30. Lewis SA, Pavord ID, Stringer JR, Knox AJ, Weiss ST, Britton JR. The relation between peripheral blood leukocyte counts and respiratory symptoms, atopy, lung function, and airway responsiveness in adults. Chest. 2001;119:105-14.

31. Boulet L-P, Lemière C, Archambault F, Carrier G, Descary MC, Deschesnes F. Smoking and asthma: clinical and radiologic features, lung function, and airway inflammation. Chest. 2006:129:661-8.

32. Telenga ED, Kerstjens HAM, Ten Hacken NHT, Postma DS, van den Berge M. Inflammation and corticosteroid responsiveness in ex-, current- and neversmoking asthmatics. BMC Pulm Med. 2013;13:58.

33. McGrath KW, Icitovic N, Boushey HA, Lazarus SC, Sutherland ER, Chinchilli VM, et al. A large subgroup of mild-to-moderate asthma is persistently noneosinophilic. Am J Respir Crit Care Med. 2012;185:612-9.

\section{Submit your next manuscript to BioMed Central and we will help you at every step:}

- We accept pre-submission inquiries

- Our selector tool helps you to find the most relevant journal

- We provide round the clock customer support

- Convenient online submission

- Thorough peer review

- Inclusion in PubMed and all major indexing services

- Maximum visibility for your research

Submit your manuscript at www.biomedcentral.com/submit

) Biomed Central 